\title{
Structural uterine anomalies in recurrent pregnancy loss
}

\author{
Rizwana Habib, Asma Hassan Mufti*, Nasir Jeelani Wani \\ Department of Obstetrics and Gynecology, Government Medical College Srinagar, Jammu and Kashmir, India
}

Received: 09 March 2019

Accepted: 02 April 2019

\section{*Correspondence:}

Dr. Asma Hassan Mufti,

E-mail: asma.h.mufti14@gmail.com

Copyright: $\odot$ the author(s), publisher and licensee Medip Academy. This is an open-access article distributed under the terms of the Creative Commons Attribution Non-Commercial License, which permits unrestricted non-commercial use, distribution, and reproduction in any medium, provided the original work is properly cited.

\begin{abstract}
Background: To determine frequency of different structural uterine anomalies in patients with recurrent pregnancy loss.

Methods: This observational study was conducted over a period of one and half year at a tertiary care Hospital, included 40 women with recurrent pregnancy loss who underwent combined laparoscopy and hysteroscopy.

Results: Twenty-eight patients $(70 \%)$ had 3 episodes of miscarriage, eight patients $(20 \%)$ had experienced 4 abortions and three patients $(7.5 \%)$ had five abortions. Only one patient $(2.5 \%)$ had six abortions. $32.5 \%$ patients had normal hysteroscopy while as $65 \%$ patients had no abnormal finding on laparoscopy. Hysteroscopy was abnormal in $67.5 \%$ patients with uterine septum $(25 \%)$ being the most common finding followed by submucous myoma(20\%), polyp (12.5\%), cervical incompetence $(7.5 \%)$ and intra uterine adhesions $(2.5 \%)$. Laparoscopy was abnormal in $35 \%$ patients with endometriosis $(17.5 \%)$ being the most common finding followed by intra pelvic adhesions $(15 \%)$ and bicornuate uterus $(2.5 \%)$.

Conclusions: Women with recurrent pregnancy loss have increased association with structural uterine anomalies than general population. Both congenital and acquired uterine anomalies are associated with recurrent abortions.
\end{abstract}

Keywords: Hysteroscopy, Laparoscopy, Recurrent pregnancy loss, Structural uterine anomalies

\section{INTRODUCTION}

Pregnancy loss, especially when recurrent is an emotionally traumatic experience. Recurrent pregnancy loss (RPL), habitual abortion or recurrent miscarriage is defined as the occurrence of three or more consecutive pregnancies that end in miscarriage of the fetus before the age of viability i.e. 20 weeks. ${ }^{1}$ About $15 \%$ of couples lose one recognized pregnancy and $2 \%$ of couples lose two. ${ }^{2}$ Studies suggest that the risk of miscarriage in subsequent pregnancies is $30 \%$ after two losses compared with $33 \%$ after three losses among patients without a history of live birth. $^{3}$ It has been reported that $1-3 \%$ of women experience recurrent loss of pregnancy and the cause is not identified in approximately $50 \%$ of cases. ${ }^{4}$ Of the identifiable causes, chromosomal disorders like translocation and aneuploidy are responsible for $2-5 \%$ cases while as anatomic factors account for $10-15 \%$ of cases.

Uterine anomalies are directly associated with recurrent pregnancy loss and can be divided into congenital and acquired forms. Congenital anomalies include fusion abnormalities or partial agenesis of developing uterus such as septate, bicornuate, didelphis or unicornuate uterus. $^{5}$

Furthermore, a number of acquired uterine anomalies such as fibroids, intrauterine adhesions, Asherman syndrome, endometrial polyps, adenomyosis, endometriosis and cervical incompetence have been associated with recurrent pregnancy loss. ${ }^{6}$ 
During development, the uterus forms via the apposition of a portion of bilateral hollow tubes called mullerian ducts. The dissolution of the walls of these ducts along their sites of apposition allows formation of intrauterine cavity, the intracervical canal and the upper vagina. Congenital uterine anomalies may therefore include incomplete mullerian duct fusion, incomplete septum and uterine cervical anomalies. Congenital uterine anomalies may arise from malformations at any step of the mullerian developmental process. ${ }^{7}$

Buttram and Gibbons first proposed a classification of the congenital uterine anomalies based on the degree of failure of the mullerian ducts to develop normally, and divided them into groups with similar clinical manifestations, treatment and prognosis. This was revised and modified first in 1983 and then in 1988 by the American society of reproductive medicine to provide a classification which is now the most widely accepted and used worldwide. This consists of seven groups, some with further subdivisions:

Mullerian agenesis or hypoplasia

- Vaginal,

- Fundal,

- Cervical,

- Tubal,

- Combined.

Unicornuate uterus (agenesis or hypoplasia of one of the two Mullerian ducts)

- With a communicating rudimentary horn

- With a non-communicating rudimentary horn

- With a rudimentary horn with no cavity

- With an absent rudimentary horn

Didelphys uterus (failure of fusion of the paired Mullerian ducts)

Bicornuate uterus (incomplete fusion of the uterine horns at the level of the fundus)

- Complete

- Partial

Septate uterus (absent or incomplete resorption of the uterovaginal septum)

- Complete

- Partial

Arcuate uterus (a mild indentation at the level of the fundus from a near complete resorption of the uterovaginal septum)

Diethylstilbestrol (DES) exposed uterus (t-shaped uterus resulting from DES exposure of the patient in utero)
Women with an intrauterine septum may have as high as $60 \%$ risk of spontaneous abortion. Uterine septum related losses most frequently occur during the second trimester, however, if an embryo implants into the poorly developed endometrium overlying the uterine septum, abnormal placentation and resultant first trimester losses may occur.

The prevalence of arcuate uterus in women with recurrent pregnancy loss is $12.2 \%$, such high prevalence suggests causal relation between arcuate uterus and recurrent pregnancy loss. ${ }^{8}$

Cervical incompetence classically causes losses in the second trimester (gestational weeks 12 to 28). It is associated with painless cervical dilatation and expulsion of the fetus. The diagnosis requires two consecutive losses. Cervical incompetence can be caused by congenital factors (e.g., DES exposure), trauma (including forceps delivery, especially if performed before full dilatation is complete), and surgical procedures (e.g., cervical cone biopsies, laser procedures, and loop electrocautery excision procedures). The chance of carrying the baby to term can be improved by placing a cervical cerclage at 10 to 14 weeks, after fetal viability has been confirmed. ${ }^{2}$

Asherman syndrome is also associated with recurrent miscarriage. ${ }^{9}$ The presence of intrauterine adhesions may significantly impact placentation and result in early pregnancy loss.

Fibroids distort the endometrial cavity and impede the implantation. Intramural fibroids larger than $5 \mathrm{~cm}$ in size as well as submucosal fibroids of any size can cause recurrent pregnancy loss.

The septate uterus is most commonly associated with reproductive failure. ${ }^{10}$ Hysterosalpingography (HSG) may reveal two hemi cavities, without visualization of uterine fundus and may be indistinguishable from a bicornuate uterus. Although HSG remains a useful tool for diagnosis of a normal or abnormal uterine cavity, it cannot reliably differentiate between different types of structural uterine anomalies and allow appropriate classification. ${ }^{11,12}$

Although 3D ultrasound has been used in diagnosis of septate uterus only a few studies are available with no definite conclusion and its role in identification of uterine septum. ${ }^{13}$ Combining diagnostic modalities can improve diagnostic accuracy but concurrent hysteroscopy and laparoscopy remains the gold standard for diagnosis of septate uterus. ${ }^{3}$

\section{METHODS}

This prospective observational study was conducted in a tertiary care hospital over a period of one and half year. 


\section{Inclusion criteria}

- Women with three or more consecutive spontaneous pregnancy losses were included in this study

\section{Exclusion criteria}

- Patients with established diagnosis of recurrent pregnancy loss due to endocrine, autoimmune or genetic causes and patients with major medical illness (liver disease, diabetes) coagulation disorders or on oral anti-coagulant therapy were excluded.

Women included were counseled and informed about the method of this study. They were subjected to detailed medical, surgical, family, obstetric and gynecological histories.

A detailed physical examination was carried out. All prior pregnancies were assessed with attention to gestational age at time of loss, complications and pathology reports, if any pelvic ultrasonography and hysterosalpingograms were accessed and evaluated.

All base line and specific investigations to rule out autoimmune, endocrine and genetic causes of recurrent pregnancy loss were done. Serum progesterone (mid luteal progesterone on day 21 of 28-day cycle) was done to rule out luteal phase defect as a cause of recurrent pregnancy loss. Serum prolactin and thyroid function tests were carried out to exclude hyperprolactinemia and thyroid function abnormalities. Follicle stimulating hormone (FSH) and luteinizing hormone (LH) on day 3 was also done. Transvaginal and transabdominal sonography was carried out before the procedure. All patients recruited for the study were admitted 2 days prior to the procedure, in post menstrual period as during early proliferative phase uterine cavity is easily evaluated. Preanaesthetic evaluation was carried out. Combined hysteroscopy and laparoscopy were carried out after written informed consent from the patient.

\section{RESULTS}

A total of 40 women with RPL had both hysteroscopy and laparoscopy performed. They ranged in age from 25 to 33 years, with a mean age of 29.6 years (Table 1).

Table 1: Age distribution of patients.

\begin{tabular}{|lll|}
\hline Age (years) & Number of patient: $(\%)$ \\
\hline $25-27$ & 9 & 22.5 \\
\hline $28-30$ & 17 & 42.5 \\
\hline $31-33$ & 14 & 35.0 \\
\hline Total & 40 & 100 \\
\hline Mean \pm SD $=29.6 \pm 2.51$ & \\
\hline
\end{tabular}

The range of number of RPL was 3 to 6 . Twenty-eight women (70\%) had 3 RPL, $8(20 \%)$ had 4 RPL whereas 3
(7.5\%) had 5 abortions. Only one patient $(2.5 \%)$ had six abortions. Therefore, authors had 137 episodes of abortions in our sample. Of these 137 episodes, 115 $(83.9 \%)$ occurred at the first, and $22(16.1 \%)$ at the second trimester (Table 2).

Table 2: Trimester wise distribution of abortions.

\begin{tabular}{|lll|}
\hline Trimester & No. of abortions & Percentage \\
\hline Trimester I & 115 & 83.9 \\
\hline Trimester II & 22 & 16.1 \\
\hline Total & 137 & 100 \\
\hline
\end{tabular}

Authors had 27(67.5\%) participants with only first, and 2 (5\%) with only second trimester RPL. The remaining 11 (27.5\%) patients had both first and second-trimester abortions. The mean number of abortions in present study was $3.4 \pm 0.75$. Hysteroscopy was abnormal in $67.5 \%$ patients whereas $32.5 \%$ patients had no abnormal finding on hysteroscopy (Table 3).

Table 3: Hysteroscopic findings among study patients.

\begin{tabular}{|lll|}
\hline Hysteroscopic findings & No. of patients & $(\%)$ \\
\hline Submucous myoma & 8 & 20.0 \\
\hline Septum & 10 & 25.0 \\
\hline Polyp & 5 & 12.5 \\
\hline Intrauterine adhesions & 1 & 2.5 \\
\hline Cervical incompetence & 3 & 7.5 \\
\hline
\end{tabular}

Septum was found in 10 patients (25\%), which was the most common finding, followed by submucous myoma present in 8 (20\%). Polyp was found in 5 patients $(12.5 \%)$ whereas 3 patients $(7.5 \%)$ had cervical incompetence. Only one patient $(2.5 \%)$ had intrauterine adhesions. Laparoscopy was normal in majority of patients i.e. $65 \%$ whereas $35 \%$ patients had abnormal laparoscopic findings with endometriosis being found in seven patients $(17.5 \%)$ whereas six patients $(15 \%)$ had intrapelvic adhesions. Only one patient $(2.5 \%)$ had a bicornuate uterus (Table 4).

Table 4: Laparoscopic findings among study patients.

\begin{tabular}{|lll|}
\hline Laparoscopic findings & No. of patients & $(\%)$ \\
\hline Endometriosis & 7 & 17.5 \\
\hline Intrapelvic adhesions & 6 & 15.0 \\
\hline Bicornuate uterus & 1 & 2.5 \\
\hline
\end{tabular}

In present study acquired conditions like submucous myoma, polyp, intrauterine adhesions, cervical incompetence, endometriosis and intrapelvic adhesions, were found in thirty patients $(73.2 \%)$ whereas eleven patients $(26.8 \%)$ had congenital uterine anomalies which included septate and bicornuate uterus (Table 5).

There were no intraoperative or postoperative complications noted in this study. 
Table 5: Endoscopic findings among study patients.

\begin{tabular}{|lll|}
\hline Endoscopic findings & Number & $(\%)$ \\
\hline Congenital & 11 & 26.8 \\
\hline Acquired & 30 & 73.2 \\
\hline
\end{tabular}

\section{DISCUSSION}

In present study, majority of the patients were in the age group of $28-30(42.5 \%)$, mean age being $29.6 \pm 2.51$ years. It was comparable to study conducted by Khameneh MK et al, in which mean age of patients was 30.4 years. ${ }^{14}$ Similar observations were made by Elbareg AM et al, who in their study found mean age of patients 28.3 years. ${ }^{15}$ Mean number of abortions in present study with recurrent pregnancy loss was 3.4 \pm 0.75 . Bohlmann MK et al, (2010) in their study found mean number of abortions as 3.74. Khameneh MK et al, in their study found mean number of abortions as 3.8 where as Elbareg AM et al, (2014) reported mean number of abortions as $2.8 \pm 0.6$ in their study. ${ }^{14,15} 115$ abortions i.e., $83.9 \%$ were first trimester abortions whereas only 22 abortions took place in second trimester which constituted only $16.1 \%$. In the study conducted by Khameneh MK et al, first trimester abortions constituted $73 \%$ and second trimester abortions accounted for $27 \%$ of total number of abortions. ${ }^{14}$ In present study hysteroscopy was abnormal in $67.5 \%$ patients where as $32.5 \%$ patients had no finding. In the study conducted by Elbareg AM et al, $59.9 \%$ of patients had normal hyteroscopy whereas $40.1 \%$ of the patients had abnormal hysteroscopic findings. ${ }^{15}$ Khameneh MK et al, reported $16 \%$ of patients had normal findings on hyteroscopy and in $84 \%$ of the patients hysteroscopy was abnormal where as normal hysteroscopy constituted $60.9 \%$ and abnormal hysteroscopy accounted for $39.1 \%$ in the study conducted by Ventolini $\mathrm{G}$ et al. ${ }^{14,16}$

In the hysteroscopic findings in present study septum was found in 10 patients $(25 \%)$, which was the most common finding, submucous myoma was present in 8 patients (20\%),polyp was found in 5 patients accounting for $12.5 \%$ of the patients, whereas 3 patients $(7.5 \%)$ had cervical incompetence. Only one patient $(2.5 \%)$ had intrauterine adhesions. In the study done by Sekin B et al, 43 patients $(16 \%)$ had a septate uterus, three patients (1\%) had a unicornuate uterus, 18 patients $(7 \%)$ had intrauterine adhesions, 17 patients $(6 \%)$ had endometrial polyps, and two patients (1\%) had a submucous myoma. ${ }^{17}$ In the study conducted by Ventolini $G$ et al, five patients $(21.8 \%)$ had intrauterine adhesions, two patients $(8.7 \%)$ had a septate uterus, one patient $(4.3 \%)$ had submucosal myoma, and one patient $(4.3 \%)$ had multiple factors. ${ }^{16}$ In the study done by Khameneh MK et al, septate uterus and submucous myomas together, comprised $62(77.5 \%)$ of the recognized pathologies in hysteroscopy. ${ }^{14}$

In present study, laparoscopy was normal in $65 \%$ patients where as $35 \%$ patients had abnormal laparoscopic findings. Similarly, in the study conducted by Khameneh
MK et al, $65 \%$ of patients had normal findings on laparoscopy whereas $35 \%$ of the patients had abnormal laparoscopic findings. ${ }^{14}$

The frequency of various laparoscopic findings in present study was endometriosis which was found in seven patients accounting for $17.5 \%$ of the patients whereas six patients $(15 \%)$ had intrapelvic adhesions and only one patient $(2.5 \%)$ had a bicornuate uterus. In the study conducted by Khameneh MK et al, $15 \%$ patients had endometriosis, whereas abdominal adhesions were present in $12 \%$ of the patients. ${ }^{14}$

In present study acquired uterine factors were found in thirty patients accounting for $73.2 \%$ of the patients whereas eleven patients $(26.8 \%)$ had congenital uterine anomalies. Similarly Sarvelos SH et al, in there study found that the prevalence of congenital uterine anomalies is approximately $16.7 \%$ (95\% CI, 14.8-18.6) in the recurrent miscarriage population while as in the study conducted by Elbareg AM et al, the congenital uterine anomalies were in $79(24.4 \%)$ and acquired in 51 (15.7\%) women. ${ }^{18,15}$

\section{CONCLUSION}

In present study hysteroscopy was abnormal in $67.5 \%$ patients with most common finding being uterine septum followed by submucous fibroid and polyp. Intra uterine adhesions and cervical incompetence were the less common findings on hysteroscopy. Laparoscopy was normal in $65 \%$ of patients whereas only $35 \%$ of patients had abnormal laparoscopic findings. Endometriosis was the most common abnormal finding on laparoscopy followed by intrapelvic adhesions and bicornuate uterus.5\% of patients had abnormal findings both on hysteroscopy and laparoscopy.Thus, concluding that women with recurrent pregnancy loss have increased association with structural uterine anomalies than general population. Both congenital and acquired uterine anomalies are associated with recurrent abortions.

\section{Funding: No funding sources Conflict of interest: None declared Ethical approval: Not required}

\section{REFERENCES}

1. American society for reproductive medicine. Evaluation and treatment of recurrent pregnancy loss: a committee opinion. Fert Steril. 2012;98:110311.

2. Kiwi R. Recurrent pregnancy loss: evaluation and discussion of the causes and their management. Cleveland Clinic J Med. 2006;73(10):913.

3. Ford HB, Schust DJ. Recurrent pregnancy loss: etiology, diagnosis, and therapy. Reviews Obstet Gynecol. 2009;2(2):76.

4. Toth B, Jeschke U, Rogenhofer N, Scholz C, Würfel W, Thaler CJ, Makrigiannakis A. Recurrent 
miscarriage: current concepts in diagnosis and treatment. J Reprod Immunol. 2010;85(1):25-32.

5. Homer HA, Li TC, Cooke ID. The septate uterus: a review of management and reproductive outcome. Fertil Steril. 2000;73(1):1-4.

6. Bettocchi S, Siristatidis C, Pontrelli G, Sardo AD, Ceci O, Nappi L, et al. The destiny of myomas: should we treat small submucous myomas in women of reproductive age?. Fertil Steril. 2008;90(4):90510.

7. Devi Wold AS, Pham N, Arici A. Anatomic factors in recurrent pregnancy loss. Semin Reprod Med. 2006;24(1):25-32.

8. Saravelos SH, Cocksedge KA, Li TC. Prevalence and diagnosis of congenital uterine anomalies in women with reproductive failure: a critical appraisal. Human Reprod Update. 2008;14(5):415-29.

9. Li TC, Makris M, Tomsu M, Tuckerman E, Laird S. Recurrent miscarriage: aetiology, management and prognosis. Human Reprod Update. 2002;8(5):46381 .

10. Taylor E, Gomel V. The uterus and fertility. Fertil Steril. 2008;89(1):1-6.

11. Ludwin A, Ludwin I, Banas $T$, Knafel A, Miedzyblocki M, Basta A. Diagnostic accuracy of sonohysterography, hysterosalpingography and diagnostic hysteroscopy in diagnosis of arcuate, septate and bicornuate uterus. J Obstet Gynaecol Res. 2011;37(3):178-6.

12. Ghi T, Casadio P, Kuleva M, Perrone AM, Savelli L, Giunchi S, et al. Accuracy of three-dimensional ultrasound in diagnosis and classification of congenital uterine anomalies. Fertil Steril. 2009;92(2):808-13.
13. Makris N, Kalmantis K, Skartados N, Papadimitriou A, Mantzaris G, Antsaklis A. Three-dimensional hysterosonography versus hysteroscopy for the detection of intracavitary uterine abnormalities. Int $\mathbf{J}$ Gynecol Obstet. 2007;97(1):6-9.

14. Khameneh MK. Combined hysteroscopy and laparoscopy in the evaluation of patients with recurrent pregnancy loss. Int Med J Malaysia. 2013;12(1) 33-38.

15. Elbareg AM, Essadi FM, Elmehashi M, Anwar KI, Adam I. Hysteroscopy in Libyan women with recurrent pregnancy loss. Sudan J Med Sci. 2014;9(4):239-44.

16. Ventolini G, Zhang M, Gruber J. Hysteroscopy in the evaluation of patients with recurrent pregnancy loss: a cohort study in a primary care population. Surg Endoscopy. 2004;18(12):1782-4.

17. Seckin B, Sarikaya E, Oruc AS, Celen S, Cicek N. Office hysteroscopic findings in patients with two, three, and four or more, consecutive miscarriages. European J Contracept Reprod Health Care. 2012;17(5):393-8.

18. Saravelos SH, Cocksedge KA, Li TC. Prevalence and diagnosis of congenital uterine anomalies in women with reproductive failure: a critical appraisal. Human Reprod Update. 2008;14(5):415-29.

Cite this article as: Habib R, Mufti $\mathrm{AH}$, Wani NJ. Structural uterine anomalies in recurrent pregnancy loss. Int J Reprod Contracept Obstet Gynecol 2019;8:2039-43. 